\title{
Aerodynamic AND Motion Study OF FLAPPING WINGS VEHICLES
}

\author{
Chawki Abdessemed ${ }^{\mathrm{a}}$, Janis Viba ${ }^{\mathrm{b}}$ \\ ${ }^{a}$ Riga Technical University, abdessemed@hotmail.com, \\ ${ }^{b}$ Riga Techical University, janis.viba@rtu.lv
}

\begin{abstract}
Micro aerial vehicles design, due to their low Reynolds number aerodynamics, motivated the aerospace engineering community a lot the last year, and especially the flapping wing operated MAVs.

The simulation of the mechanical equations of motion for a flapping wing MAV is presented in this work to have an approximate the behavior and the condition of flight of the vehicle and to present a control model that can be implemented to auto control the vehicle. The spherical coordinates system is used to develop the equations in this work and Mathcad software is mainly used for the solution, simulation and graphing of the results, constants related to the size of the vehicle are changed to match different a range of existing flying insects or birds. Upstroke and down stroke of the flapping wing were modeled using two different drag coefficient. The study resulted in excluding the smaller sizes and higher flapping frequency from use of this model without any rectifications that account for the accentuated mow Reynolds unsteady effect, but bigger vehicles (bird sized) were modeled with a good accuracy and the model can be used as well for auto-control by predefined flying path. A small experimentation on a model wing covered in feather was conducted in a subsonic wind tunnel in order to present a practical alternative that economize energy by reducing the drag in upstroke.
\end{abstract}

Keyword: Aerodynamic, control, equation of motion, feather, flapping, MAV, wings.
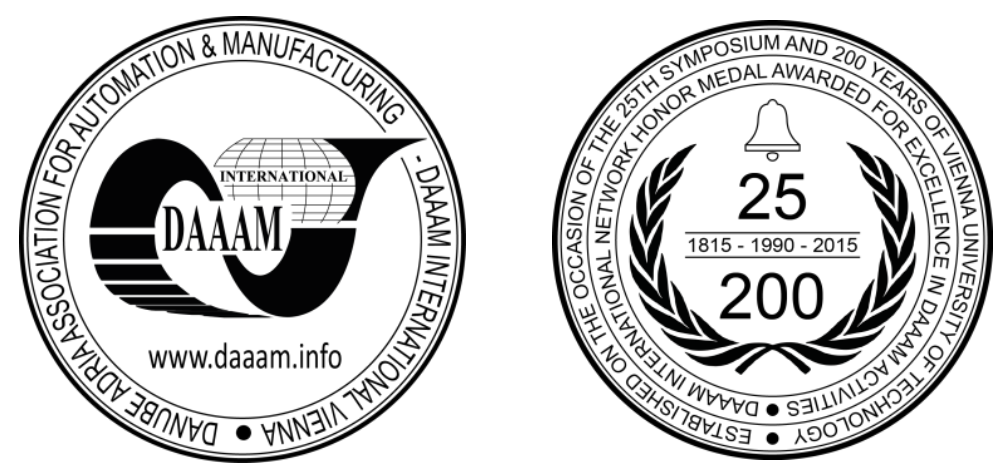

This Publication has to be referred as: Abdessemed, A[hmed] C[hawki] \& Viba, J[anis] (2016). Aerodynamic and Motion Study of Flapping Wings Vehicle, Proceedings of the 26th DAAAM International Symposium, pp.0834-0841, B. Katalinic (Ed.), Published by DAAAM International, ISBN 978-3-902734-07-5, ISSN 1726-9679, Vienna, Austria DOI:10.2507/26th.daaam.proceedings.116 


\section{Introduction}

Birds, insect and bats was a subject of fascination to the human since the dawn of humanity, and he did not just content by envying their ability, but he invested his energy and intellect in order to mimic them and conquer the sky using the ability of those great flyers.

However it was only recently with the advent of simulation, analysis and high-speed videography that we could decipher some of their complex mechanisms that make them that good.

Flapping wings vehicles have many secrets for the engineers, such a way of generating lift and thrust was examined closely from pioneers from Abbas Ibn Firnas to Leonardo di Vinci.

However, researchers are more and more attracted by this kind of propulsion for application on a small scale flapping wings vehicles, because of the need to a smaller, manoeuvrable aerial vehicle.

DARPA (Defense Advanced Research Projects Agency) [1] encouraged the research and design of such vehicles and initiated the MAVs project which set its major goal the solving of the major technical barrier that prevent from the realizations of such machine.

\section{State of art in micro aerial vehicle design}

\subsection{Definition of MAV:}

The MAV was originally defined as a vehicle with a maximal dimension of $15 \mathrm{~cm}$ or less, which is comparable to the size of small birds and a flight speed of $10-20 \mathrm{~m} / \mathrm{s}$ they are equipped with sensors or a video camera and a gross take-off weight of no more than $100 \mathrm{~g}$.

This physical size puts this class of vehicle at least an order of magnitude smaller than any missionized UAV developed to date.

Four types of configurations [2] exist in this category: fixed-wing, rotary-wing, and flapping wings, the fourth class is without propulsion and is called passive. since the dawn of aviation the first 2 categories did benefit a lot from the studies conducted, but due to the lack of manoeuvrability for the first one which requires a forward speed to fly and cannot hover, the second one have efficiency problems, and this due to the flow physics in this regime. That is why the focus on the third category, which is the flapping wings.

\subsection{Flapping wings:}

Nature makes it clear that this propulsion mechanism is very efficient and effective in an aerodynamics view at a low Reynolds number and in the same time allows high degrees of manoeuvrability, humming birds are the best example, while despite requiring a lift coefficient, in the quasi-steady sense, over twice that of any aircraft. Bumblebees are able to fly. However, a distinction should be made when focusing on this class of vehicles between bird-like vehicles called Ornithopter and Insect-like vehicles called Entemopters [1].

Ornithopter fly more like fixed-wing aircraft, they require forward speed to fly and generate lift by flapping wings up and down with synchronized small variations of angle of incidence. Entemopters (insect like) attempt to imitate insect Insects' wing kinematics [3] which uses large and rapid change in wing angle of incidence at the end of each stroke. The last ones suits more MAV tasks because hovering and this class provides manoeuvre in tight spaces. a complete different aerodynamic effects in flight is used compared to the rotary machine for instance, which still make use of the steady aerodynamic principles however the entemopters rely on fully unsteady aerodynamics to fly

The advantages offered by flapping wing vehicles seduced the engineers to pursue in this direction come up with an efficient and effective vehicle, but the MAV are quite tiny compared to the challenges. Despite the enormous challenges, last years experienced advance in the MAV field where several promising MAVs, like the one developed by Festo Company revealed, the SmartBird (Fig.1). Other promising MAV was revealed in March 2013, probably the most advanced and ambitious MAV constructed until those words are written, the BionicOpter with this dragonfly-like AV (fig.3) a little bit outside of the range to be called MAV due to its $63 \mathrm{~cm}$ wing span and $44 \mathrm{~cm}$ long, probably the most amazing thing about it is its 13 degree of freedom, More details about other more or less developed MAV can be found in [8] and [9].

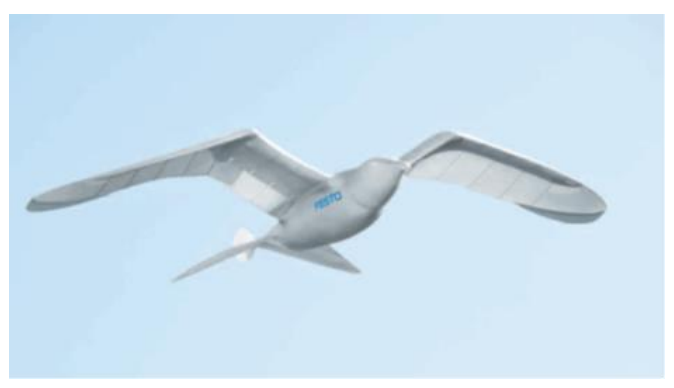

Fig.1. Festo’s SmartBird. 


\section{Statement of Work:}

The simulation of the mechanical equations of motion for a flapping wing MAV is the focus of this work, which results in the approximate behavior and condition of flight of a flapping wing vehicle and to present a control model that can be implemented to auto control the vehicle.

Some variables were changed while others were kept constant and approached value for the drag coefficient of birds and insects were used, this was done in the spherical coordinates system. The same equations permit to create a control system that can be implemented electronically to steer the MAV. The whole technique provides results that have a good accuracy and have many advantages such as:

1. A very good approximation about the flight parameter.

2. It allows a significant economy in the time of calculation and the cost of computer memory and money comparing to a direct numerical simulation.

3. It a control method that can steer the MAV and guide it using some criteria of stability.

An experimental works was be conducted in the wind tunnel facility provided by Riga technical University, which consisted of testing a wing's drag coefficient and its variation between the upstroke and the down stroke.

\section{Numerical investigation with Mathcad}

\subsection{Theoretical investigation:}

The flight of the vehicle is well described my Newtonian mechanics, the equations of equilibrium which result from Newton's second law are used to describe the flapping wing vehicle, and other systems of coordinates than the rectangular is used because it describes better this motion in space [9]. The resultant of all the forces acting on a particle is proportional to the acceleration of the particle:

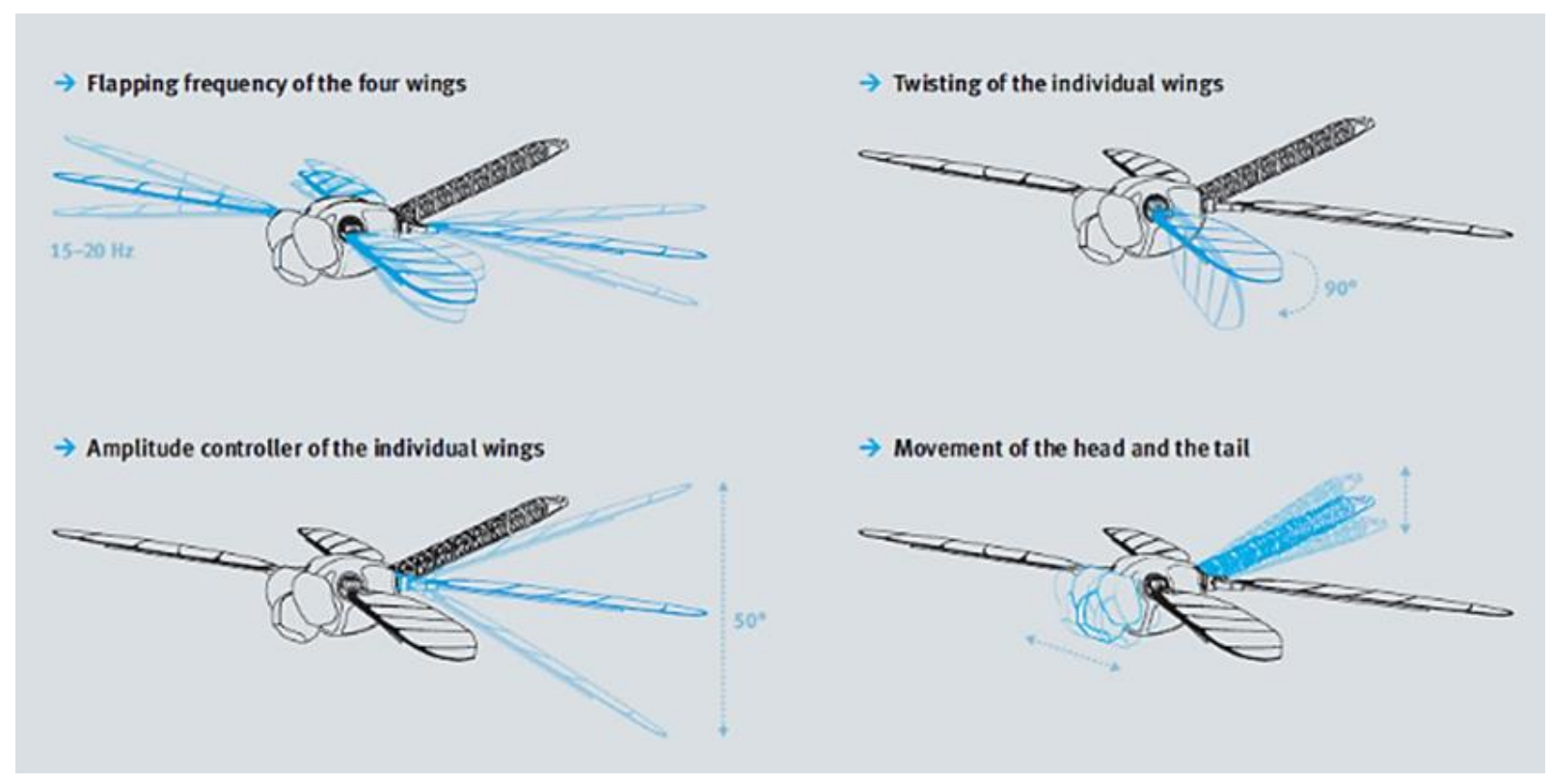

Fig. 2. Some Characteristic of the BionicOpter.

$\sum F=m a$

By applying this basic principal to a body in motion in a plan, the equations in a rectangular basis would be:

$\sum F_{x}=m a_{x}$

$\sum F_{y}=m a_{y}$

\subsection{Spherical Coordinates $(r-\theta-\varphi)$ :}

Two angles are used in addition to a distance to specify the position of a particle. 


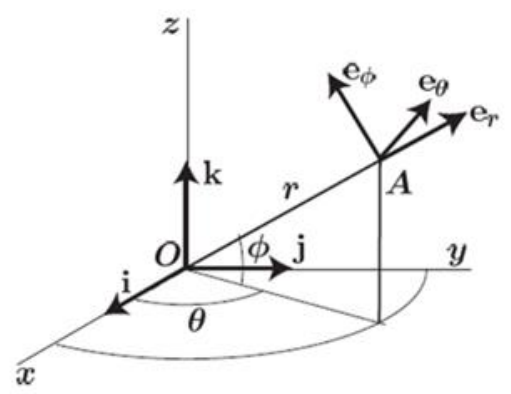

Fig.3: spherical Coordinate

The acceleration is:

$\mathrm{a}=\mathrm{a}_{\mathrm{r}} e_{r}+\mathrm{a}_{\theta} e_{\theta}+\mathrm{a}_{\varphi} e_{\varphi}$

Therefore the equations of motion in 3D spherical coordinates are:

$$
\begin{aligned}
& \sum F_{r}=m a_{r}=m\left(\ddot{r}-r \dot{\theta}^{2} \cos \varphi^{2}-r \dot{\varphi}^{2}\right) \\
& \sum F_{\theta}=m a_{\theta}=m(2 \dot{r} \dot{\theta} \cos \varphi-r \ddot{\theta} \cos \varphi-2 r \dot{\theta} \dot{\varphi} \sin \varphi) \\
& \sum F_{\varphi}=m a_{\varphi}=m\left(2 \dot{r} \dot{\varphi}+r \dot{\varphi}^{2} \sin \varphi \cos \varphi+r \ddot{\varphi}\right)
\end{aligned}
$$

\section{Application on a real case:}

Despite their simplicity the previous equations of motion as can be used in order to simulate the flight of a flapping wing MAV; Mathcad was used with an increment in time in order to simulate the flight. However, we need to define the forces acting on the MAV.

\subsection{Lift force}

The original idea of this work [9] is the use of two drag coefficient in the expression of the resultant force ; as we deduct from nature, when a wing is in the upstroke the muscle is used to alter the shape of the wing to cause less drag therefore a smaller drag coefficient $\mathrm{C}_{\mathrm{d} 1}$, in contrast in the down stroke the shape of the wing is optimal for a large lift force which is the opposite of the drag force, if we change the direction of the axis, therefore a biggest drag coefficient $\mathrm{C}_{\mathrm{d} 2}$.

With the introduction of this two-drag coefficient approach, we will have two forces (Fig. 4) and knowing that the lift force expression is:

$F_{L}=\frac{1}{2} \rho C_{L} A V^{2}$

Where:

$\rho:$ density of the air.

$\mathrm{V}$ : the velocity.

$\mathrm{S}$ : the lifting area (both wings).

$\mathrm{C}_{\mathrm{L}}$ : lift coefficient

By introducing both drags coefficients:

$F_{1}=\frac{1}{2} \rho C_{d 1} A V^{2}$ and $F_{2}=\frac{1}{2} \rho C_{d 2} A V^{2}$

The switch between the two different drag coefficients in the upstroke and the down stroke is done by the introduction of a formulate that uses the direction (therefore sign) of the velocity the deactivate one or the other coefficient such as:

$0.5-0.5 \operatorname{sign}(\cos (\omega t)), 0.5+0.5(\cos (\omega t))$ 
The velocity approximated from the sinusoidal motion of the wing, the path would be A. sin $(\omega)$ therefore the velocity can be expressed as the first derivative: $A \cdot \omega \cdot \cos (\omega t)$ another sign related expression is needed so the expression of the velocity is:

$$
V^{2}=(A \omega \cos (\omega t))^{2} \operatorname{sign}(\cos (\omega t))
$$

Where:

$\omega$ : Angular frequency.

A: Amplitude.

Therefore, the expression of the lift force is as following:

$F=\frac{1}{2}\left(\left(0.5-0.5 \operatorname{sign}(\cos (\omega t)) C_{d 1}+0.5+0.5 \operatorname{sign}(\cos (\omega t)) C_{d 2}\right) \cdot S \rho(A \omega \cos (\omega t))^{2} \cdot \operatorname{sign}(\cos (\omega t))\right.$

\subsection{Damping force:}

We should consider as well the damping force giving the harmonic "vibration" and its expression is:

$$
F_{d}=-b \frac{d x}{d t}
$$

A projection of all those expressions on their respective axis is performed to find the 3D equations.

For The radial, coordinate axis:

$$
\begin{aligned}
& \ddot{r}=\frac{1}{m}\left(-m g \sin \varphi+m r \dot{\theta}^{2} \cos ^{2} \varphi+m r \varphi^{2}+\left[\frac{1}{2}\left(\left(0.5-0.5 \operatorname{sign}(\cos (\omega t)) C_{d 1}+0.5+0.5 \operatorname{sign}(\cos (\omega t)) C_{d 2}\right) \cdot S \cdot \rho \cdot(A \omega \cos (\omega t))^{2} \operatorname{sign}(\cos (\omega t))\right] .\right.\right. \\
& \left.\cos \beta \cos (\varphi-\alpha)-b \cdot \frac{d r}{d t}\right)
\end{aligned}
$$

For The polar axis:

$$
\begin{aligned}
& \ddot{\theta}=\frac{1}{m \cdot r \cdot \cos \varphi}(-2 m \dot{r} \theta \cos \varphi+2 m \dot{\theta} \dot{\varphi} \sin \varphi)+\left[\frac { 1 } { 2 } \left(\left(0.5-0.5 \operatorname{sign}(\cos (\omega t)) C_{d 1}+0.5+0.5 \operatorname{sign}(\cos (\omega t)) C_{d 2}\right) .\right.\right. \\
& \text { S. } \left..(A \omega \cos (\omega t))^{2} \operatorname{sign}(\cos (\omega t))\right] \cdot \cos \beta \sin \left(\frac{\pi}{2}+\varphi-\alpha\right) \\
& \left.- \text { b.r. } \cos (\varphi) \frac{d \theta}{d t}\right)
\end{aligned}
$$

The Azimuth coordinate axis:

$$
\begin{aligned}
& \ddot{\varphi}=\frac{1}{m}\left(-m g \cdot \cos \varphi-2 m \dot{r} \dot{\varphi}-m r \dot{\varphi}^{2} \sin \varphi \cos \varphi+\left[\frac { 1 } { 2 } \left(\left(0.5-0.5 \operatorname{sign}(\cos (\omega t)) C_{d 1}+0.5+\right.\right.\right.\right. \\
& \left.\left.0.5 \operatorname{sign}(\cos (\omega t)) C_{d 2}\right) \cdot S \cdot \rho \cdot(A \omega \cos (\omega t))^{2}\right] \cdot \sin (\beta)-b . r \cdot \frac{d \varphi}{d t}
\end{aligned}
$$

A certain criteria was added the last equation in order to control the altitude of the MAV, [9]:

$$
\left(0.5-0.5 . \operatorname{sign}\left(\cos \left(r \cos \varphi-H_{m}\right)\right)\right.
$$

\section{Mathcad implementation}

Two different range of dimension were used, the bird -like and insect-like, in the literature we found the parameters needed and a formula developed by PENNYCUICK, that link the flapping frequency of the birds wing to the other parameter ( $\mathrm{m}, \mathrm{S}, \rho, \mathrm{g}, \mathrm{b}$ (wing span)) [6] was uses, which is written as:

$$
f=m^{3 / 8} g^{1 / 2} b^{-23 / 24} S^{-1 / 3} \rho^{-3 / 8}
$$


Where:

$\mathrm{m}$ : is the body mass.

$\mathrm{g}$ is the acceleration due to gravity.

$\mathrm{b}$ is the wingspan.

$\mathrm{S}$ : is the wing area.

$\rho:$ is the air density.

Therefore, the expression of the Angular frequency is:

$\omega=2 \pi \cdot m^{3 / 8} g^{1 / 2} b^{-23 / 24} S^{-1 / 3} \rho^{-3 / 8}$

For the bird-like configuration two different sizes were used, the first ones from DARPA [9] definition the second one from an actual bird which is the Dove prion. For the insect-like configuration, dimensions of a dragonfly were used.

\subsection{Drag coefficient:}

It is mentioned in the literature that the lift to drag ratio is between 3 and 17 which gives a drag coefficient for birds between 0.8 and 1.2 however the motion of the wing affect significantly the coefficient therefore a set of drag coefficient around the real value were used .For insect like MAVs insects wing have very small drag coefficient around 0.06 , so we used numbers around it.

\section{Mathcad manipulations}

The Cartesian coordinates where obtained by the transformations:

$$
\begin{aligned}
& x=r \cos \varphi \sin \theta \\
& y=r \sin \phi \sin \theta \\
& z=r \cos \theta
\end{aligned}
$$

We found out that the MAV goes very high up to 40m, which means that the force is very strong; therefore, changes in the damping coefficient were done for the radial coordinates, by steps of 0.05 . For the first step: b1=0.05 we obtained the data in Graph1. [1]

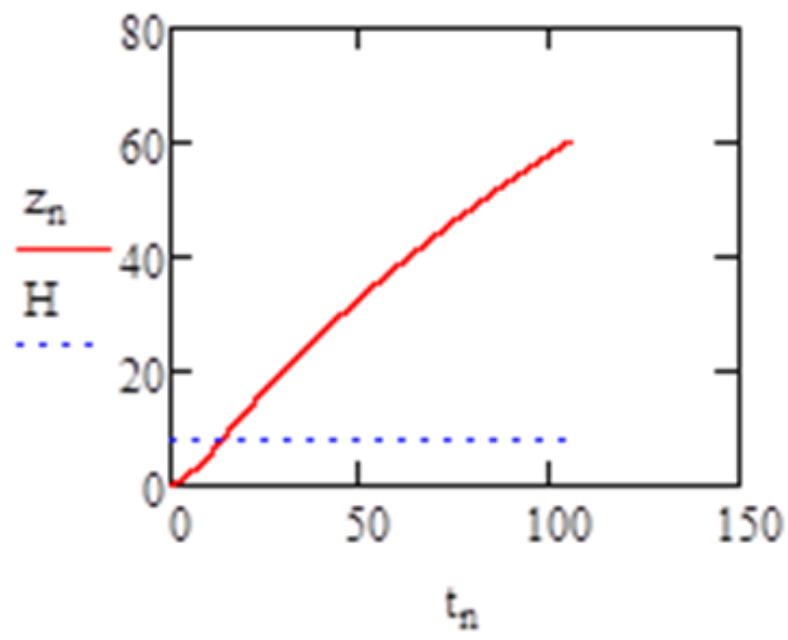

Fig. 4. We noticed at the drag coefficient value of 1.8 the MAV starts to fly but in a very non-stable way.

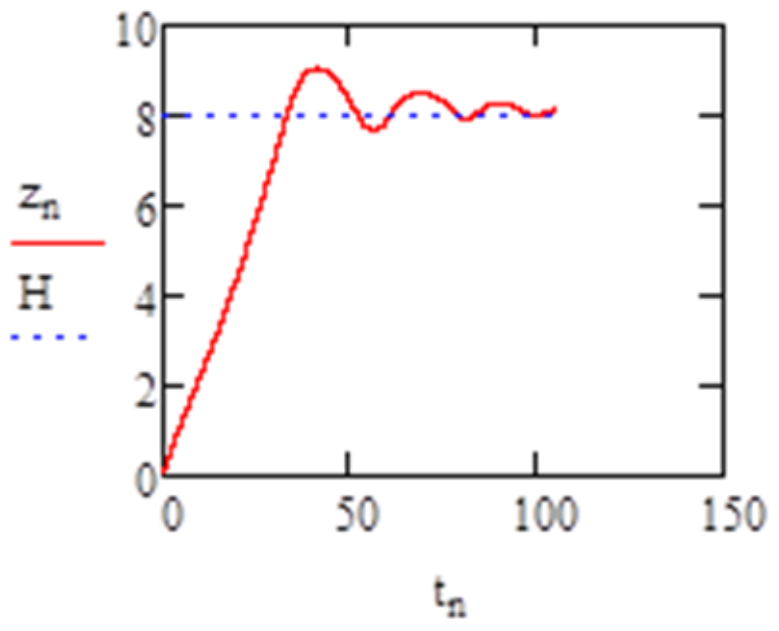

Fig. 5. Vertical coordinates in the spherical case.

The criteria works and the MAV find stability in a while the results shown in graphs 4,5 . We performed more manipulations on different parameters [7] the results are illustrated in graphs (3), (4), (5) It was obvious the instability occurred in the $\mathrm{z}$-axis so the damping coefficient was changed from $\mathrm{b}$ to $\mathrm{b} 1$ and the later was varied. the increase of the damping coefficient ( $b 1=0.1$ and 0.15$)$ the results were close but far more stable, as it's illustrated in the graphs (8) where we can see the path of flying in the $(r, \phi)$ 


\subsection{Steering}

We steered the MAV by directly implementing and fixing the angles $\alpha$ and $\beta$ or by making one or both of the angles time dependent. We used as an example [7] the change in angles value by a time depending functions:

$$
\begin{aligned}
& \alpha=\frac{\pi}{2.5} \cdot\left(e^{-0.01 t}\right) \\
& \beta=1.5 \cdot \frac{\pi}{2}\left(e^{-0.01 t}\right)
\end{aligned}
$$

This do not affect the vertical coordinates; however, we can see the change of path in the other plans In the Graph 8 we see that after the stabilization the MAV stop turning in circles and take a fixed direction.

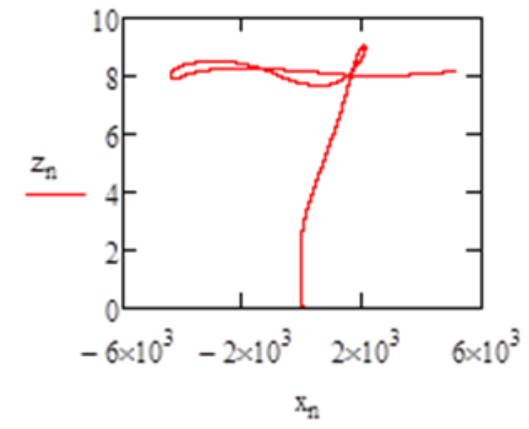

Fig. 6. The flight path in $(\mathrm{x}, \mathrm{z})$ plan $\mathrm{b} 1=0.05$.

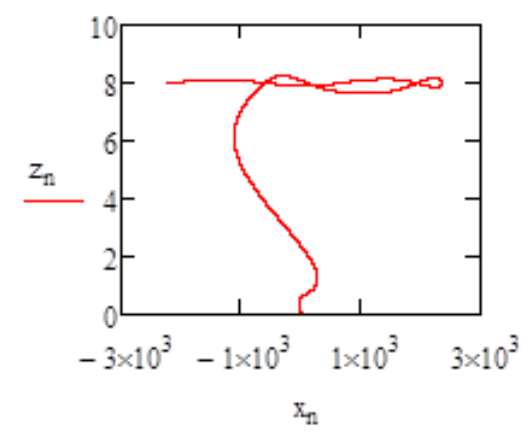

Fig. 8. The flight path in $(\mathrm{x}, \mathrm{z})$ plan

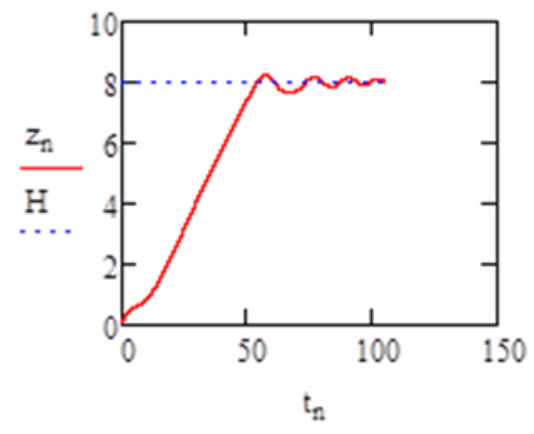

Fig. 7. The vertical coordinates $b 1=0.1$

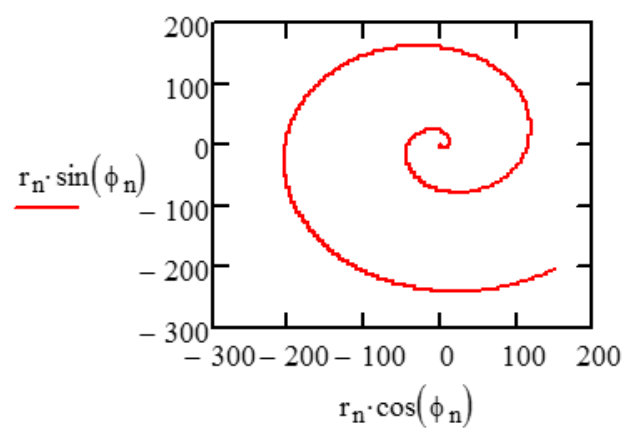

Fig. 9. The flight path in $(\mathrm{x}, \mathrm{y})$ plan as helicoid

\subsection{DARPA definition MAV}

We tried the DARPA definition $\mathrm{m}=100 \mathrm{~g}$, wing span $\mathrm{bs}=15 \mathrm{~cm}$. along with a range of area and drag coefficients. we noticed that if we use the frequency formula with smaller areas ,the frequency resulting will be too high and nonrealistic, in the other hand with bigger area the MAV fly perfectly with realistic flapping frequency, but the wing ratio will be too high.

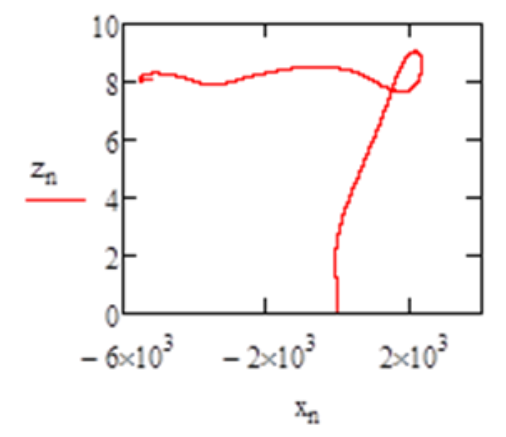

Fig. 10. Effect of steering (x,z) plan..

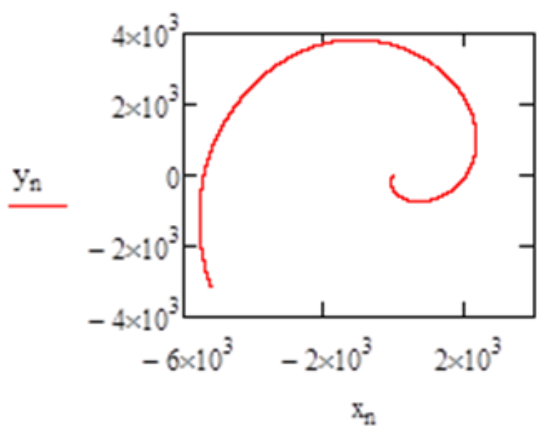

Fig. 11. Effect of steering $(\mathrm{x}, \mathrm{y})$ plan.

\subsection{Dimensions of an insect}

We tried the characteristics of a dragonfly, we did some manipulation in all the parameter but the MAV only flies for very high frequency, amplitude, or drag, so this model does not work for very small dimensions. 


\section{Experimental Work}

It consists simply of measuring the drag force that is produced by an artificial pair of wing covered with feather (Fig. 8) from both sides, and we tried to see if the profile's camber as well as the feather orientation affects the drag.

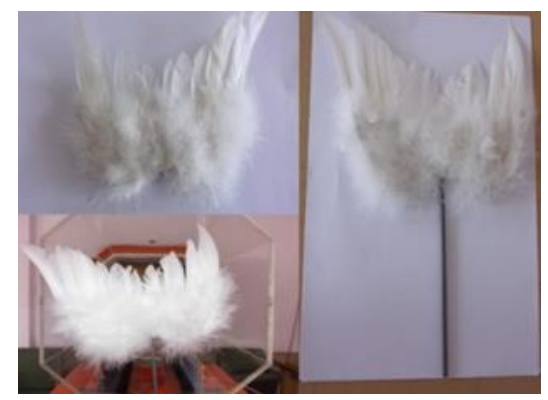

Fig. 12. The tested artificial wing.

Results are illustrated in graph 9

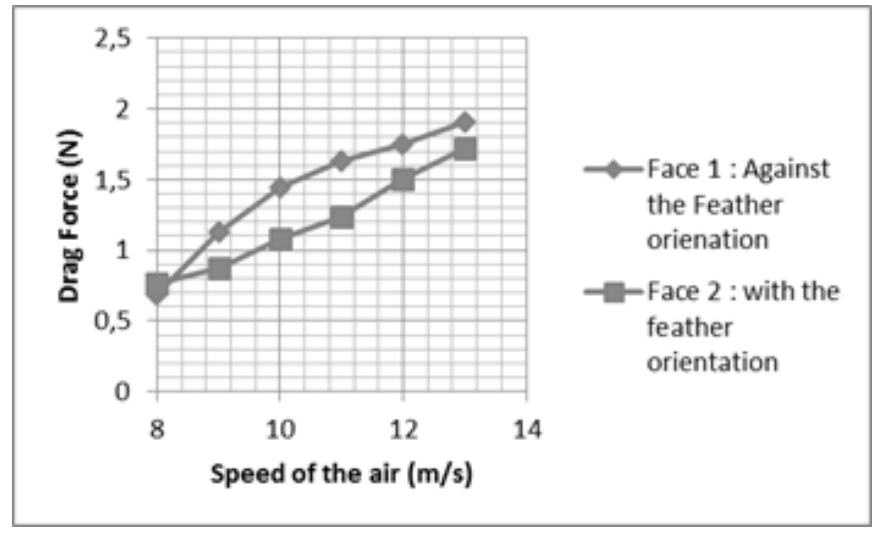

\section{Results}

Fig. 13. Graph that illustrates the result of the measurements performed on the wing

We can summarize the results in the next points:

1. The major parameter that influences the MAV flying is the damping coefficients.

2. The flapping frequencies have to be defined and limited under certain value to keep up with the reality, and the available force for flapping.

3. The difference between the two drags coefficients plays a central role in making the MAV fly.

4. And finally, we had a confirmation that due to the unsteady aerodynamic phenomenon that are accentuated in the insect case ,the approximation used cannot be applied.

\section{References}

[1] L. Petricca, P. Ohlckers, and C. Grinde, "Micro- and Nano-air vehicles: state of the art", International journal of aerospace engineering, vol. 2011, no: 1, 2011.

[2] Al-Bahi, i.m.a.-q.a.a.m., "Micro Aerial Vehicles Design Challenges: State Of The Art Review", in SSAS UAV Scientific Meeting \& Exhibition. 2006: jeddah, Saudi Arabia.

[3] M. James and C. M. S. F. McMichael, "Micro Air Vehicles-Toward a New Dimension in Flight, 1997.

[4] T. J. Mueller, "Aerodynamic measurements at low Reynolds numbers for fixed wing micro air vehicles", Tech. Rep., University of Notre Dame, Notre Dame, The Netherlands, 2000.

[5] http://www.festo.com/cms/en_corp/13165.htm

[6] C. J. Pennycuick, 'Wing beat Frequency of Birds in Steady Cruising Flight: New Data and Improved Predictions", The Journal Of Experimental Biology 199, 1613-1618, 1996.

[7] A.C.Abdessemed, "Investigation Of The Flight Of A Micro Aerial Vehicle", Master thesis, Riga Technical University,2013.

[8] W.Shyy a,d al, "An Introduction to Flapping Wing Aerodynamics" 2013.

[9] A.C.Abdessemed, Janis Viba, "Investigation of flight of micro aerial vehicle with flapping wings", Proceedings of 13th International Scientific Conference Engineering For Rural Development.2014. E-Publishing Inc., New York, 1999, pp. 281-304. 